\title{
Mitofusin 1 degradation is induced by a disruptor of mitochondrial calcium homeostasis, CGP37157: A role in apoptosis in prostate cancer cells
}

\author{
VIVEK CHOUDHARY ${ }^{1-3}$, ISMAIL KADDOUR-DJEBBAR $^{1-3}$, RABEI ALAISAMI $^{3}$, \\ M. VIJAY KUMAR ${ }^{1,2,4}$ and WENDY B. BOLLAG ${ }^{1,3}$ \\ ${ }^{1}$ Charlie Norwood VA Medical Center; ${ }^{2}$ Section of Urology, Department of Surgery, ${ }^{3}$ Department of Physiology, \\ Medical College of Georgia at Georgia Regents University, Augusta, GA, USA \\ Received January 5, 2014; Accepted February 24, 2014
}

DOI: $10.3892 /$ ijo.2014.2343

\begin{abstract}
Mitochondria constantly divide (mitochondrial fission) and fuse (mitochondrial fusion) in a normal cell. Disturbances in the balance between these two physiological processes may lead to cell dysfunction or to cell death. Induction of cell death is the prime goal of prostate cancer chemotherapy. Our previous study demonstrated that androgens increase the expression of a mitochondrial protein involved in fission and facilitate an apoptotic response to CGP37157 (CGP), an inhibitor of mitochondrial calcium efflux, in prostate cancer cells. However, the regulation and role of mitochondrial fusion proteins in the death of these cells have not been examined. Therefore, our objective was to investigate the effect of CGP on a key mitochondrial fusion protein, mitofusin 1 (Mfn1), and the role of Mfn1 in prostate cancer cell apoptosis. We used various prostate cancer cell lines and western blot analysis, qRT-PCR, siRNA, M30 apoptosis assay and immunoprecipitation techniques to determine mechanisms regulating Mfn1. Treatment of prostate cancer cells with CGP resulted in selective degradation of Mfn1. Mfn1 ubiquitination was detected following immunoprecipitation of overexpressed Myc-tagged Mfn1 protein from CGP-treated cells, and treatment with the proteasomal inhibitor lactacystin, as well as siRNA-mediated knockdown of the E3 ubiquitin ligase March5, protected Mfn1 from CGP-induced degradation. These data indicate the involvement of the ubiquitin-proteasome pathway in CGP-induced degradation of Mfn1. We also demonstrated that downregulation of Mfn1 by siRNA enhanced the
\end{abstract}

Correspondence to: Dr Wendy B. Bollag, Department of Physiology, Medical College of Georgia at Georgia Regents University, 1120 15th Street, Augusta, GA 30912, USA

E-mail:wb@gru.edu

Present address: ${ }^{4}$ Southern Arizona VA Health Care System, 3601 S 6th Avenue, Tucson, AZ 85723, USA

Key words: mitochondrial fusion, mitofusin 1, ubiquitination, prostate cancer, apoptosis apoptotic response of LNCaP cells to CGP, suggesting a likely pro-survival role for Mfn1 in these cells. Our results suggest that manipulation of mitofusins may provide a novel therapeutic advantage in treating prostate cancer.

\section{Introduction}

Prostate cancer is the second leading cause of cancer death among men in the United States, with an estimated 30,000 deaths among American men in 2014 (American Cancer Society, www.cancer.org). For effective treatment of prostate cancer it is necessary to identify effective and specific molecular targets. As cancer cells exhibit various degrees of mitochondrial dysfunction (1-4), it is of interest to determine whether therapeutic strategies focused on mitochondria can be developed to preferentially kill cancer cells.

Mitochondria are dynamic organelles that play essential roles in cellular metabolism, homeostasis and regulation of cell death. Increasing evidence has demonstrated that changes in mitochondrial morphology are important determinants of mitochondrial function $(1,5)$. Mitochondria range from long filamentous structures to small punctate spheres in different cell types and under different conditions in the same cell type $(6,7)$. Mitochondrial structure is affected by the interplay between the opposing fission and fusion processes that occur normally in a cell (8). Formation of extensive mitochondrial networks (generated by fusion) is considered important for efficient intracellular energy transfer into different cell compartments (9), whereas the punctate mitochondria (arising from fission) seem to assist, in some cases, the induction of apoptosis (10-14).

Mitochondrial fission involves the large GTPase, dynamin-related protein 1 (Drp1) and fission 1 (Fis1) (1). Recent discoveries suggest that mitochondrial fission factor (Mff), MIEF1/MiD51 and Mid49 act as novel mitochondrial receptors for Drp1 to regulate mitochondrial dynamics in mammalian cells (15-19). Mitochondrial fusion of the outer membranes is controlled by mitofusin 1 (Mfn1) and mitofusin 2 (Mfn2) whereas optic atrophy 1 (OPA1) is implicated in fusion of inner mitochondrial membranes $(20,21)$. Mitofusins are large transmembrane GTPases of the mitochondrial outer membrane, which facilitate the fusion of these membranes in 
a GTPase-dependent manner (1,22-25). Overexpression of Mfn1 results in the formation of characteristic networks of interconnected mitochondria (24), whereas loss of mitofusin function causes mitochondrial fragmentation and dysfunction $(1,24,26-28)$. In the nervous system, overexpression of Mfn1 can protect against NO-induced neuronal cell death (29). In yeast, it has been shown that Mdm30p ligase promotes ubiquitination of Fzolp, a yeast homologue of mammalian Mfn1, and its subsequent degradation by the $26 \mathrm{~S}$ proteasome $(30,31)$. Recently, March5, an E3 ubiquitin ligase, has been shown to be involved in mammalian Mfn1 degradation in HeLa and Chang cells (32).

In recent years, agents that impact mitochondria and exhibit anticancer activity are gaining attention as possible cancer therapies. CGP37157 (CGP) inhibits the efflux of mitochondrial calcium through the sodium/calcium exchanger, thereby increasing calcium levels in mitochondria and altering their function. Earlier, we demonstrated that CGP treatment increases the interaction between Drp1 and Fis1 to induce mitochondrial fission (14), while CGP when combined with TNF- $\alpha$ related apoptosis-inducing ligand (TRAIL), sensitizes TRAIL-resistant prostate cancer cells to undergo apoptosis (33). We have reported that androgen upregulates the expression and levels of Drp1, a protein involved in mitochondrial fission, but does not by itself induce mitochondrial fission or apoptosis (11). However, treatment of LNCaP cells with CGP in the presence of androgen increases mitochondrial fission and apoptosis (11), suggesting that the androgen-induced increase in Drp1 is not sufficient to trigger mitochondrial fission and apoptosis but requires a second signal to affect mitochondrial function and enhance these processes. Although we demonstrated a concomitant decrease in the fusion protein Mfn1, the mechanism by which CGP affected Mfn1 protein expression was not investigated. Therefore, our objective was to investigate the regulation of the mitochondrial fusion protein, Mfn1, in response to CGP treatment as well as its potential role in prostate cancer cell apoptosis.

\section{Materials and methods}

Reagents. The mammalian Myc-tagged Mfn1 overexpression plasmid (Myc-Mfn1; a kind gift from Alexander van der Bliek, University of California, Los Angeles, CA) was used for ubiquitination studies. Antibodies used were: anti-Mfn1 (Novus Biologicals, Littleton, CO); anti-Mfn2, anti- $\beta$-actin and anti-chicken IgG (Sigma-Aldrich Corporation, St. Louis, MO); anti-GAPDH (Chemicon International, Temecula, CA); anti-COX IV and anti-mouse and anti-rabbit IgG (Cell Signaling, Danvers, MA); anti-Drp1 (BD Biosciences, San Jose, CA); antiubiquitin and anti-Fis1 (Santa Cruz Biotechnology, Santa Cruz, CA); and anti-March5 and anti-OPA1 (Abcam Inc., Cambridge, MA). The chemicals used were: cycloheximide (SigmaAldrich); 7-chloro-5-(2-chlorophenyl)-1,5-dihydro-4,1-benzothiazepin-2(3H)-one or CGP37157 (CGP) (Calbiochem, San Diego, CA); and lactacystin (Cayman Chemical Company, Ann Arbor, MI). Scrambled siRNA and siRNA for Mfn1 and March5 were from Qiagen (Valencia, CA). The c-Myc immunoprecipitation kit was from Thermo Scientific (Rockford, IL).

Cell cultures and treatment. Prostate cancer cell lines LNCaP, DU145, and PC3 were purchased from ATCC (Manassas, VA).
Cells were maintained in RPMI-1640 (HyClone, Logan, UT) containing $9 \%$ FBS (v/v), $0.5 \%$ penicillin-streptomycin (v/v), and $0.1 \%$ fungizone (v/v). The CWR-R 1 cells (provided by Dr Elizabeth Wilson, University of North Carolina, Chapel Hill, NC) were grown in Richter's MEM. Non-tumorigenic prostate epithelial cells (P69, a gift from Dr Leland Chung) were maintained in T-medium (Gibco-Invitrogen, Carlsbad, CA). Cells were treated with cycloheximide $(50 \mu \mathrm{g} / \mathrm{ml})$ or CGP $(10-80 \mu \mathrm{M})$ for the indicated period of time. Transfection with siRNA was performed using HiPer-Fect reagent (Qiagen) for $48 \mathrm{~h}$ followed by treatment with various drugs. Cells were transiently transfected with the myc-tagged Mfn1 overexpression plasmid (Myc-Mfn1) by electroporation using the Electro Square Porator ${ }^{\mathrm{TM}}$ ECM-830 (BTX Inc., San Diego, CA) as described previously (11). After electroporation, cells were seeded in normal growth medium for $24 \mathrm{~h}$ and then used in experiments.

Protein extraction and western blot analysis. Appropriately treated cells were washed once with 1X PBS followed by addition of lysis buffer $[150 \mathrm{mM} \mathrm{NaCl}, 50 \mathrm{mM}$ Tris- $\mathrm{HCl}$, $\mathrm{pH}$ 8.0, 1 mM EDTA, $0.7 \%$ (v/v) Triton X-100, 0.25\% (w/v) sodium deoxycholate, $1 \mathrm{mM} \mathrm{NaF}, 1 \mathrm{mM}$ sodium pyrophosphate, $100 \mu \mathrm{M} \mathrm{Na}_{3} \mathrm{VO}_{4}, 1 \mathrm{mM}$ phenylmethylsulfonyl fluoride, $10 \mu \mathrm{g} / \mathrm{ml}$ leupeptin, $0.7 \mu \mathrm{g} / \mathrm{ml}$ pepstatin, and $10 \mu \mathrm{g} / \mathrm{ml}$ aprotinin] at $4^{\circ} \mathrm{C}$. Cells were incubated on ice for $30 \mathrm{~min}$ and lysates centrifuged at $10,000 \mathrm{x} \mathrm{g}$ at $4^{\circ} \mathrm{C}$ for $10 \mathrm{~min}$. The supernatants were collected, and the protein concentration was estimated using a Bio-Rad DC protein reagent (Bio-Rad Laboratories, Hercules, CA). Proteins $(25 \mu \mathrm{g})$ were separated on 10 or $12 \%$ (w/v) SDS-polyacrylamide gels and transferred to (PVDF) membranes (Bio-Rad Laboratories) using a BioRad semi-dry transfer apparatus. The blots were blocked in 5\% (w/v) non-fat dry milk in TBS containing $0.1 \%$ (v/v) Tween-20 and incubated overnight at $4{ }^{\circ} \mathrm{C}$ with primary antibody. Immunoreactive bands were visualized using an ECL detection system (Amersham, Pharmacia Biotech, Arlington Heights, IL) on an 8900 Alpha Innotech Image Analyzer (San Leandro, CA) and/or exposed to Hyperfilm and developed. The membranes were re-probed with antibody against $\beta$-actin and/or GAPDH, which were used as loading controls. Antibodies were diluted in $3 \%(\mathrm{w} / \mathrm{v})$ BSA in TBST.

Measurement of apoptosis. Measurement of apoptosis was performed using an M30 Apoptosense kit (Peviva, DiaPharma Group, West Chester, $\mathrm{OH}$ ) as described previously (11). Briefly, total cell lysates $(5 \mu \mathrm{g})$ were added to 96 -well plates pre-coated with mouse monoclonal M30 antibody; horseradish peroxidase tracer solution was then added to the wells and incubated for $4 \mathrm{~h}$. Color was developed by adding tetramethyl benzidine solution and the optical density was measured at $450 \mathrm{~nm}$ on a Spectra MAX 340 microplate reader (Molecular Devices Corporation, Sunnyvale, CA). Standard curves were generated as instructed by the supplier.

Statistical analyses. Data are presented as means \pm SEM. We compared group mean values, as appropriate, using one-way analysis of variance with Newman-Keuls multiple comparison test (GraphPad Prism). Significant differences were defined at $\mathrm{p}<0.01$ and $\mathrm{p}<0.05$. 

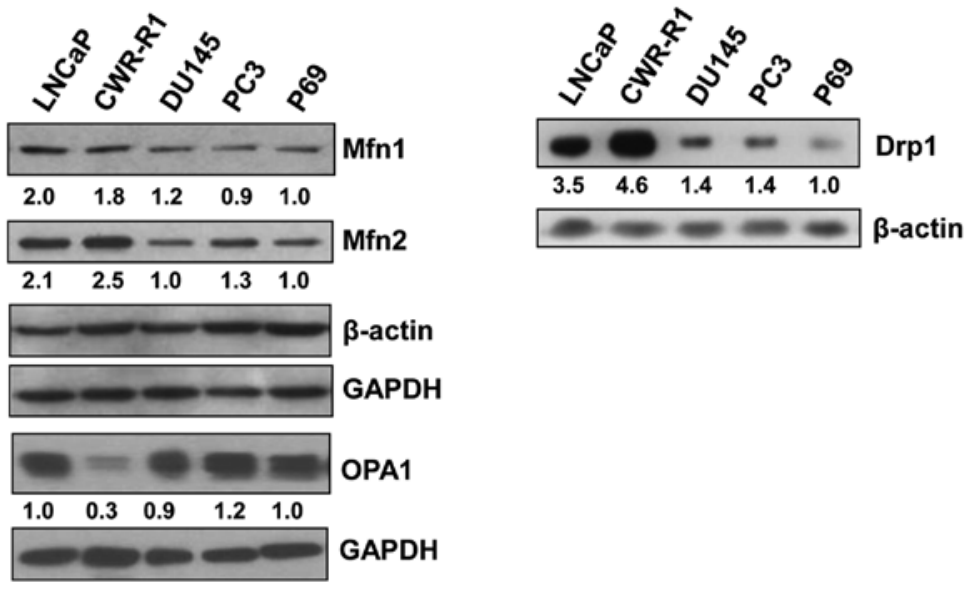

Figure 1. Basal levels of mitochondrial fusion and fission proteins in prostate cancer cell lines. Total cell lysates ( $25 \mu \mathrm{g}$ ) from the indicated prostate cancer cells were analyzed by western blotting using specific antibodies against Mfn1, Mfn2, Opa1 and Drp1. $\beta$-actin and/or GAPDH were used as loading controls, and the value (or the average value) of their optical density was used for normalization in the densitometric analysis of mitochondrial protein levels. The numbers below the bands indicate the fold difference of normalized densitometric values of that treatment group when compared to that of the normal prostate cell line, p69. The results are representative of at least three separate experiments, except the blot for Opal, which is the representative of two separate experiments.

A

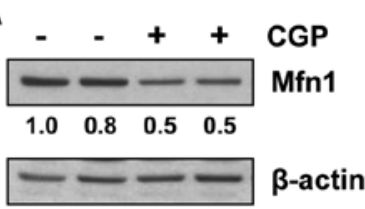

B

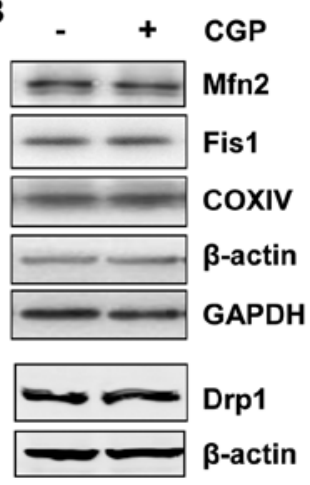

C

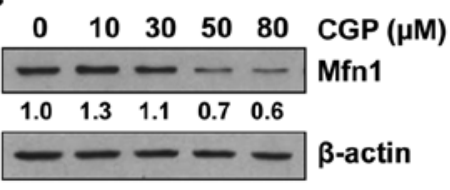

D

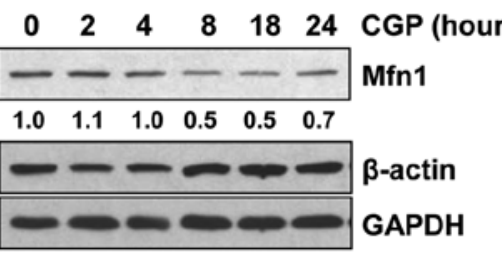

E

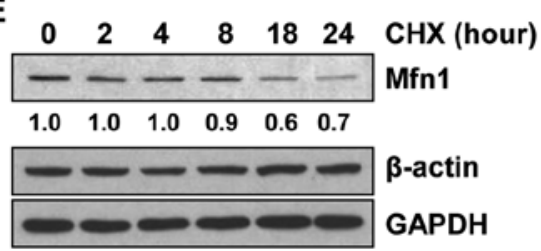

$\mathbf{F}$
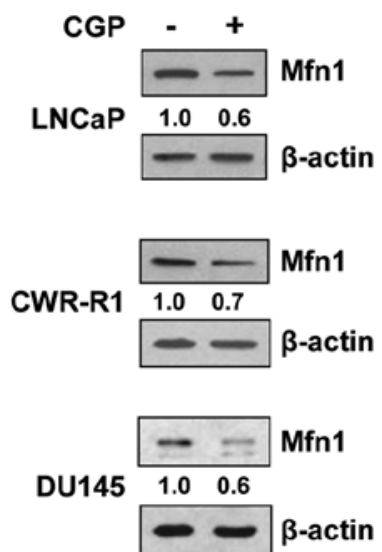

Figure 2. CGP treatment resulted in a decrease in Mfn1 protein levels. (A) LNCaP cells were treated with CGP (50 $\mu \mathrm{M})$ for $18 \mathrm{~h}$ and total cell lysates $(25 \mu \mathrm{g})$ were analyzed by western blotting using antibodies against Mfn1 and $\beta$-actin. (B) LNCaP cells were treated with CGP (50 $\mu \mathrm{M})$ for $18 \mathrm{~h}$ and analyzed by western blotting using antibodies against Mfn2, Fis1, Drp1, CoxIV, $\beta$-actin and GAPDH. (C) LNCaP cells were treated with increasing concentrations of CGP for $18 \mathrm{~h}$ and analyzed for Mfn1 protein expression by western blotting. (D) LNCaP cells were treated with CGP (50 $\mu \mathrm{M})$ for different periods of time as indicated and analyzed for Mfn1 protein expression by western blotting. (F) LNCaP cells were treated with cycloheximide (CHX; $50 \mu \mathrm{g} / \mathrm{ml})$ for different time periods, and expression of Mfn1 protein was analyzed by western blotting. Densitometric values were normalized using either a single endogenous loading control or the average of the two indicated loading controls, and the number below the bands indicates the fold difference in densitometric values of that treatment group when compared to the value of the respective control group. (E) LNCaP, CWR-R1 and DU145 cells were treated and analyzed as in (A). All the results are representative of at least three separate experiments.

\section{Results}

Basal levels of mitofusin 1 in prostate cancer cells. Western blot analysis to examine the basal levels of the proteins that regulate mitochondrial fusion in prostate cancer cell lines showed that the mitofusins, Mfn1 and Mfn2, which regulate outer mitochondrial membrane fusion, were greater in androgen receptor-positive
LNCaP and CWR-R1 cell lines as compared to androgen receptor-negative DU145 and PC3 cell lines or the normal prostate epithelial cell line, p69 (Fig. 1, left panel). Interestingly, Drp1, a protein involved in mitochondrial fission, was also higher in LNCaP and CWR-R1 cell lines (Fig. 1, right panel) compared to the other cell lines studied, suggesting that both fusion- and fission-related proteins are expressed at similar levels in these 
A

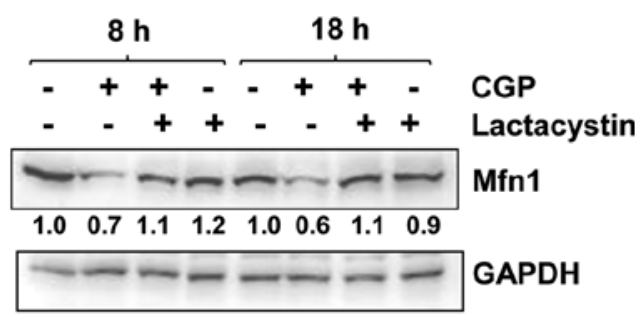

B

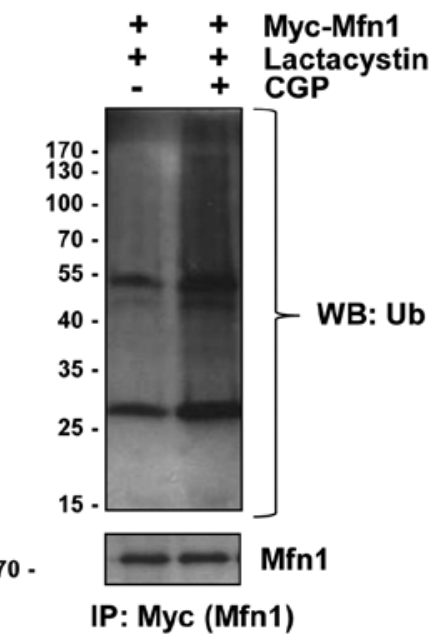

C

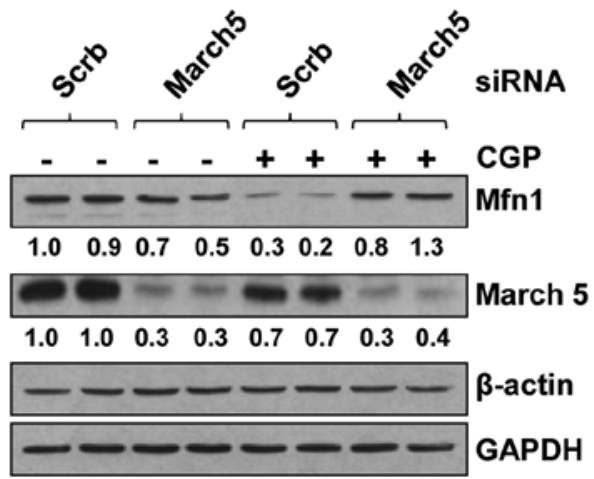

D

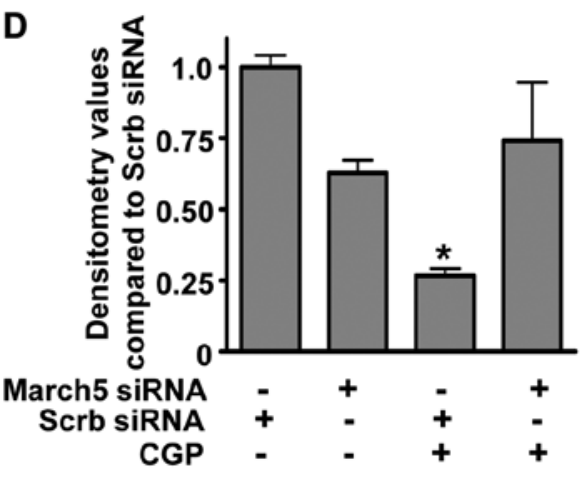

Figure 3. Mfn1 is degraded by the ubiquitin-proteasome pathway upon CGP treatment. (A) LNCaP cells were treated with or without CGP (50 $\mu \mathrm{M})$ for 8 and $18 \mathrm{~h}$ in the presence or absence of the proteasomal inhibitor, lactacystin (added $1 \mathrm{~h}$ prior to CGP and then with CGP; $5 \mu \mathrm{M}$ ). Total cell lysates ( $25 \mu \mathrm{g}$ ) were analyzed by western blotting using antibodies against Mfn1 and GAPDH. The results are representative of three independent experiments. (B) LNCaP cells were transfected with a Myc-tagged Mfn1 overexpression plasmid, pretreated with lactacystin for $1 \mathrm{~h}$ and then treated with CGP or DMSO (vehicle control) in the continued presence of lactacystin for another $8 \mathrm{~h}$. Cell lysates were subjected to immunoprecipitation with an anti-myc antibody and then analyzed by western blotting, probing first with anti-ubiquitin and then with anti-Mfn1 antibodies. The results are representative of two separate experiments. (C) LNCaP cells were transfected with March5 siRNA or scrambled (Scrb) siRNA and treated with CGP (50 $\mu \mathrm{M}$ for $8 \mathrm{~h})$. Cell lysates were analyzed by western blotting using antibodies against Mfn1, March5, $\beta$-actin and GAPDH. Shown is a representative western blot analysis from three independent experiments. (D) Cumulative densitometric analyses of multiple western blots as in $(\mathrm{C})$. The values represent the mean values \pm SEM from three independent experiments normalized using an average value for GAPDH and $\beta$-actin as endogenous loading controls. " $p<0.05$ vs. scrambled siRNA without CGP as well as March5 siRNA \pm CGP. The number below the bands indicates the fold difference in densitometric values, normalized to the loading controls, of that treatment group when compared to the value of the respective control group.

cell lines, presumably to maintain an equilibrium between the proteins having opposite roles in the regulation of mitochondrial morphology and function. The levels of OPA1, which regulates inner mitochondrial membrane fusion, were essentially similar in all cell lines except in the CWR-R1 cell line which showed a markedly lower OPA1 level (Fig. 1, left panel).

Mfnl is degraded upon CGP treatment. In previous reports we have shown that CGP sensitizes prostate cancer cells, including the LNCaP and DU145 cell line, to TRAIL-induced apoptosis (33) and induces mitochondrial fission $(11,14)$. To understand the mechanism of CGP action in the induction of mitochondrial fission, we examined the levels of Mfn1, Mfn2, Fis1 and Drp1 in LNCaP cells treated with CGP. The levels of Mfn1 protein decreased significantly (approximately 50\%) in CGP-treated LNCaP cells (Fig. 2A). However, the expression levels of the other fusion protein, Mfn2, and the fission proteins Fis1 and Drp1, were unaltered after CGP treatment of LNCaP cells (Fig. 2B). As LNCaP cells were treated with $50 \mu \mathrm{M}$ CGP in the earlier experiment (Fig. 2A), LNCaP cells were treated with increasing concentrations of CGP (up to $80 \mu \mathrm{M}$ ) to obtain a dose curve. Western blot analysis showed decreases in Mfn1 protein only when cells were treated with 50 or $80 \mu \mathrm{M}$ CGP (Fig. 2C). As LNCaP cells were treated for $18 \mathrm{~h}$ in the earlier experiments, a time course was determined by treating these cells with the minimal effective dose of $50 \mu \mathrm{M}$ CGP for various time periods up to $24 \mathrm{~h}$. A marked decrease in Mfn1 was observed after $8 \mathrm{~h}$ of CGP treatment (Fig. 2D), which was not further altered with treatment for up to $24 \mathrm{~h}$; Mfn2 levels remained largely unaltered (data not shown), consistent with results shown in Fig. 2B. Treatment of LNCaP cells with an inhibitor of protein synthesis, cycloheximide, resulted in a decrease in the levels of Mfn 1 at about $18 \mathrm{~h}$ of treatment (Fig. 2E), whereas CGP induced a reduction in Mfn1 levels as early as $8 \mathrm{~h}$ after treatment, suggesting that the decrease in Mfn1 levels in CGP-treated cells is due to degradation rather than changes in transcription/translation. CGP treatment of other cell lines (DU145 and CWR-R1) also resulted in a decrease in Mfn1 protein levels (Fig. 2F), suggesting that the ability of CGP to induce Mfn1 degradation may be common to prostate cancer cells in general.

Mfnl is degraded by a ubiquitin-proteasome pathway involving the E3 ubiquitin ligase, March5. To investigate whether 


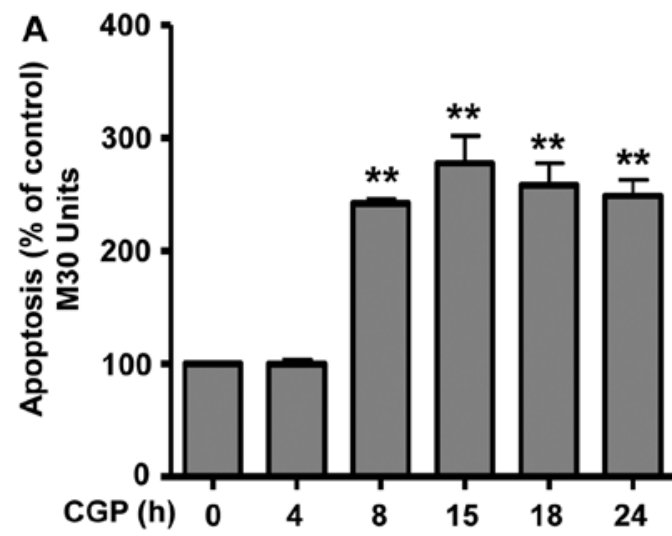

\section{B}

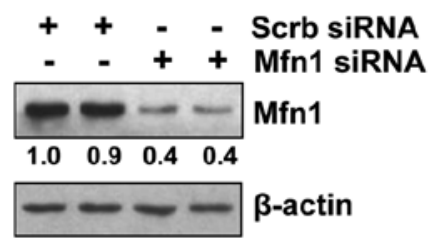

C

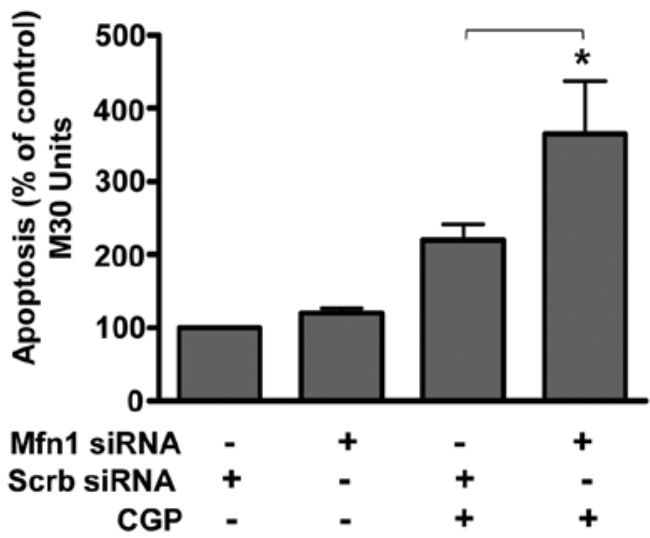

Figure 4. Degradation of Mfn1 sensitizes prostate cancer LNCaP cells to CGP-induced apoptosis. (A) LNCaP cells were treated with CGP (50 $\mu$ M) for the indicated time period. Cell lysates were collected for an apoptosis assay using the M30 apoptosense analysis kit. Results are presented as the percentage of apoptosis compared to the DMSO-treated control. The results are cumulative from at least three independent experiments. ${ }^{* *}$ p $<0.01$ vs. zero time point. (B) Cells were transfected with scrambled (Scrb) siRNA or siRNA targeting Mfn1. Cell lysates were analyzed by western blotting using antibodies against Mfn1 and $\beta$-actin. The results are representative of three separate experiments. The numbers below the bands indicate the fold difference in the densitometric values, normalized to the $\beta$-actin loading control, when compared to the value of scrambled siRNA control group in lane 1. (C) Cells were transfected with scrambled (Scrb) siRNA or siRNA targeting Mfn1 and then treated with CGP (50 $\mu \mathrm{M}$ for $8 \mathrm{~h})$. Cell lysates were used for an apoptosis assay using the M30 apoptosense analysis kit. Results are presented as the percentage of apoptosis compared to the DMSO-treated scrambled siRNA-transfected control. The results are cumulative from at least three independent experiments. " $\mathrm{p}<0.05$ vs. scrambled siRNA treated with CGP.

CGP-induced Mfn1 degradation was mediated by proteasomes, LNCaP cells were pretreated with the proteasome inhibitor, lactacystin. Pretreatment with lactacystin rescued Mfn1 from CGP-induced degradation (Fig. 3A), suggesting the involvement of proteasomes in CGP-induced Mfn1 degradation. As ubiquitination is a key step in the proteasomal degradation of a protein, we investigated whether Mfn1 is ubiquitinated upon CGP treatment. LNCaP cells were transfected with the Myc-tagged Mfn1 overexpression plasmid and treated with CGP. Proteins were immunoprecipitated using anti-Myc antibody and subjected to western blot analysis with an anti-ubiquitin antibody, which revealed that Mfn1 was ubiquitinated in CGP-treated cells (Fig. 3B). These results support our hypothesis that treatment with CGP results in ubiquitination of Mfn1, which is then targeted for degradation by proteasomes. As the E3 ubiquitin ligase, March5, has been reported to be involved in the degradation of mammalian Mfn1 protein in HeLa cervical cancer and Chang liver cells (32), its role in CGP-induced degradation of Mfn1 was examined. LNCaP cells were transfected with siRNA against March5, which significantly reduced March5 protein expression (Fig. 3C and D). Treatment of transfected cells (March5 siRNA) with CGP resulted in Mfn1 levels that were similar to the scrambled siRNA-transfected controls (Fig. 3C and D), indicating the involvement of March5 E3 ubiquitin ligase in the ubiquitination and degradation of Mfn1 upon CGP treatment.

Degradation of Mfnl sensitizes prostate cancer LNCaP cells to $C G P$-induced apoptosis. Earlier, we reported that combining CGP with TRAIL enhanced the apoptotic response to TRAIL in prostate cancer cells $(14,33)$. In these previous studies cells were treated with CGP alone or in combination with TRAIL for $4 \mathrm{~h}$; however, we did not examine the apoptotic effect of CGP alone over time. Therefore, LNCaP cells were treated with CGP for 4 to $24 \mathrm{~h}$ and apoptosis was measured. Treatment with CGP for $4 \mathrm{~h}$ did not induce apoptosis (Fig. 4A) confirming our earlier findings $(14,33)$. However, treatment with CGP for $8 \mathrm{~h}$ induced significant apoptosis. Prolonged treatment up to $24 \mathrm{~h}$ did not increase the apoptotic response, which was similar to that observed after an $8 \mathrm{~h}$ treatment (Fig. 4A). As Mfn1 was degraded upon CGP treatment within this same time frame (Fig. 2A), we hypothesized that Mfn1 degradation sensitized prostate cancer cells to CGP resulting in apoptosis. To test this hypothesis, the expression of Mfn1 was decreased by transfecting cells with Mfn1 siRNA (Fig. 4B) and then also treating with CGP. Results demonstrated a significant increase in CGP-induced apoptosis in cells expressing decreased levels of Mfn1 (Fig. 4C), suggesting that low levels of Mfn1 sensitized 
prostate cancer cells to CGP-induced apoptosis. In summary, we have demonstrated that the mitofusin Mfn1 may play a key role in the response of prostate cancer cells to apoptosisinducing agents such as CGP. Treatment with CGP decreased the levels of Mfn1 through a process involving ubiquitination via the E3 ligase March5 and proteasomal degradation. When the levels of Mfn1 were decreased, cells were readily induced to undergo apoptosis.

\section{Discussion}

Mitochondrial dysfunction has been linked to cancer (1-4), and manipulation of mitochondrial structure and function is gaining importance in the field of cancer therapeutics (11,14,34-36). We reported previously that alteration of mitochondrial function by CGP significantly increased the apoptotic response to TRAIL in TRAIL-resistant prostate cancer cells (33) and that CGP can induce mitochondrial fission in these cells (14). We have also shown that increased expression of Drp1, a protein that is known to induce mitochondrial fission and alter the function of mitochondria, induces apoptosis in these cells (14). Here, we expand our previous findings and show that reducing the levels of the mitofusin Mfn1, a protein that is involved in mitochondrial fusion to oppose the action of Drp1, sensitized prostate cancer cells to apoptotic agents, such as CGP.

The mitochondrial fusion and fission proteins play a critical role in the normal functioning of a cell. It is being increasingly appreciated that a delicate equilibrium is maintained between these proteins with opposing effects. Any alteration in the levels of these proteins may result in malfunctioning of the mitochondria $(37,38)$. Our previous observations and the data presented here concur with the above hypothesis. It is interesting to note that the expression of Mfn1 and Drp1 complemented each other. For example, cells expressing higher levels of Mfn1 (LNCaP and CWR-R1) expressed higher levels of Drp1, while those expressing lower levels of Mfn1 showed low levels of Drp1 (Fig. 1). These observations argue for the likely importance of a balance between mitochondrial fission and fusion proteins in the survival of prostate cancer cells.

There are several mechanisms described for regulation of the mitochondrial fission protein Drp1 (11,39-41). On the other hand, degradation seems to be an important mechanism for regulating the function of mitochondrial fusion proteins. Several studies reported Mfn1 degradation in yeast $(31,42)$, as well as in mammals $(32,43,44)$. Our results are consistent with the above observations in that treatment with CGP resulted in degradation of the mitochondrial fusion protein, Mfn1. Our results showed that CGP treatment resulted in ubiquitination of Mfn1 proteins leading to degradation by proteasomes. Subsequent experiments demonstrated the involvement of March5, a mitochondrial E3 ubiquitin ligase (Fig. 3C). These results agree with published data that showed that March5 is required for the degradation of Mfn1 in other cell types (32). However, we found no change in the apoptotic response of the cells to CGP when the expression of March5 was knocked down using siRNA (data not shown), possibly due to the fact that March5 is also involved in the regulation of the mitochondrial fission protein Drp1 (45). Thus, decreased degradation and increased levels of Mfn1 in cells expressing low levels of March5 could potentially be compensated for by elevations in Drp1. This duality emphasizes the complex interactions that regulate the response of the cells to apoptosis-promoting and -inhibiting proteins. The above results clearly emphasize the potential importance of mitochondrial fusion and fission proteins in devising therapeutic options. Although it is intuitive to manipulate proteins such as Drp1 involved in mitochondrial fission leading to apoptosis, it is equally critical to pay attention to the function of mitochondrial fusion proteins, such as Mfn1. Here, the enhancement of the apoptotic response to CGP in cells with reduced Mfn1 expression suggests that Mfn1 plays a pro-survival role in prostate cancer cells, although the mechanisms involved in this function require further investigation. Our results are supported by the observations that under hypoxic conditions, Mfn1 protects cancer cells from the apoptotic stimuli, staurosporine and etoposide (46), and inhibition of mitochondrial fission protects the heart against ischemia/reperfusion injury (47). Thus, manipulating mitochondrial fusion and fission machinery in prostate cancer cells may provide a new avenue of treatment for the disease.

\section{Acknowledgements}

We thank Dr Alexander van der Bliek, University of California, for providing us the Myc-Mfn1 plasmid. We also thank Dr Elizabeth Wilson and Dr Leland Chung for providing us the CWR-R1 and P69 cell lines, respectively. This research was funded by VA Merit Awards to M.V.K. and W.B.B. and an award from the American Legion of Georgia to both M.V.K. and W.B.B. The contents of this article do not represent the views of the Department of Veterans Affairs or the United States Government.

\section{References}

1. Chen G, Wang F, Trachootham D and Huang P: Preferential killing of cancer cells with mitochondrial dysfunction by natural compounds. Mitochondrion 10: 614-625, 2010.

2. Frezza C, Pollard PJ and Gottlieb E: Inborn and acquired metabolic defects in cancer. J Mol Med (Berl) 89: 213-220, 2011.

3. Kuznetsov AV, Margreiter R, Amberger A, Saks V and Grimm M: Changes in mitochondrial redox state, membrane potential and calcium precede mitochondrial dysfunction in doxorubicin-induced cell death. Biochim Biophys Acta 1813: 1144-1152, 2011.

4. Willers IM and Cuezva JM: Post-transcriptional regulation of the mitochondrial $\mathrm{H}(+)-\mathrm{ATP}$ synthase: a key regulator of the metabolic phenotype in cancer. Biochim Biophys Acta 1807: 543-551, 2010.

5. McBride HM, Neuspiel M and Wasiak S: Mitochondria: more than just a powerhouse. Curr Biol 16: R551-R560, 2006.

6. Bereiter-Hahn J and Voth M: Dynamics of mitochondria in living cells: shape changes, dislocations, fusion, and fission of mitochondria. Microsc Res Tech 27: 198-219, 1994.

7. Griparic L and van der Bliek AM: The many shapes of mitochondrial membranes. Traffic 2: 235-244, 2001.

8. Bossy-Wetzel E, Barsoum MJ, Godzik A, Schwarzenbacher R and Lipton SA: Mitochondrial fission in apoptosis, neurodegeneration and aging. Curr Opin Cell Biol 15: 706-716, 2003.

9. Skulachev VP: Mitochondrial filaments and clusters as intracellular power-transmitting cables. Trends Biochem Sci 26: 23-29, 2001.

10. Brooks C, Wei Q, Cho SG and Dong Z: Regulation of mitochondrial dynamics in acute kidney injury in cell culture and rodent models. J Clin Invest 119: 1275-1285, 2009.

11. Choudhary V, Kaddour-Djebbar I, Lakshmikanthan V, et al: Novel role of androgens in mitochondrial fission and apoptosis. Mol Cancer Res 9: 1067-1077, 2011. 
12. Frank S, Gaume B, Bergmann-Leitner ES, et al: The role of dynamin-related protein 1 , a mediator of mitochondrial fission, in apoptosis. Dev Cell 1: 515-525, 2001.

13. Gomez-Lazaro M, Bonekamp NA, Galindo MF, Jordan J and Schrader M: 6-Hydroxydopamine (6-OHDA) induces Drp1dependent mitochondrial fragmentation in SH-SY5Y cells. Free Radic Biol Med 44: 1960-1969, 2008.

14. Kaddour-Djebbar I, Choudhary V, Brooks C, et al: Specific mitochondrial calcium overload induces mitochondrial fission in prostate cancer cells. Int J Oncol 36: 1437-1444, 2010.

15. Gandre-Babbe S and van der Bliek AM: The novel tail-anchored membrane protein Mff controls mitochondrial and peroxisomal fission in mammalian cells. Mol Biol Cell 19: 2402-2412, 2008.

16. Loson OC, Song Z, Chen H and Chan DC: Fis1, Mff, MiD49, and MiD51 mediate Drp1 recruitment in mitochondrial fission. Mol Biol Cell 24: 659-667, 2013.

17. Otera H, Wang C, Cleland MM, et al: Mff is an essential factor for mitochondrial recruitment of Drp1 during mitochondrial fission in mammalian cells. J Cell Biol 191: 1141-1158, 2010.

18. Palmer CS, Osellame LD, Laine D, Koutsopoulos OS Frazier AE and Ryan MT: MiD49 and MiD51, new components of the mitochondrial fission machinery. EMBO Rep 12 $565-573,2011$

19. Zhao J, Liu T, Jin S, et al: Human MIEF1 recruits Drp1 to mitochondrial outer membranes and promotes mitochondrial fusion rather than fission. EMBO J 30: 2762-2778, 2011.

20. Cipolat S, Martins de Brito O, Dal Zilio B and Scorrano L: OPA1 requires mitofusin 1 to promote mitochondrial fusion. Proc Natl Acad Sci USA 101: 15927-15932, 2004

21. Song Z, Ghochani M, McCaffery JM, Frey TG and Chan DC: Mitofusins and OPA1 mediate sequential steps in mitochondrial membrane fusion. Mol Biol Cell 20: 3525-3532, 2009.

22. Ishihara N, Eura Y and Mihara K: Mitofusin 1 and 2 play distinct roles in mitochondrial fusion reactions via GTPase activity. J Cell Sci 117: 6535-6546, 2004.

23. Rojo M, Legros F, Chateau D and Lombes A: Membrane topology and mitochondrial targeting of mitofusins, ubiquitous mammalian homologs of the transmembrane GTPase Fzo. J Cell Sci 115: 1663-1674, 2002.

24. Santel A, Frank S, Gaume B, Herrler M, Youle RJ and Fuller MT: Mitofusin-1 protein is a generally expressed mediator of mitochondrial fusion in mammalian cells. J Cell Sci 116: 2763-2774 2003

25. Santel A and Fuller MT: Control of mitochondrial morphology by a human mitofusin. J Cell Sci 114: 867-874, 2001.

26. Eura Y, Ishihara N, Yokota S and Mihara K: Two mitofusin proteins, mammalian homologues of FZO, with distinct functions are both required for mitochondrial fusion. J Biochem 134: 333-344, 2003

27. Honda S, Aihara T, Hontani M, Okubo K and Hirose S: Mutational analysis of action of mitochondrial fusion factor mitofusin-2. J Cell Sci 118: 3153-3161, 2005.

28. Yuan H, Gerencser AA, Liot G, et al: Mitochondrial fission is an upstream and required event for bax foci formation in response to nitric oxide in cortical neurons. Cell Death Differ 14: 462-471, 2007

29. Barsoum MJ, Yuan H, Gerencser AA, et al: Nitric oxideinduced mitochondrial fission is regulated by dynamin-related GTPases in neurons. EMBO J 25: 3900-3911, 2006.

30. Cohen MM, Amiott EA, Day AR, et al: Sequential requirements for the GTPase domain of the mitofusin Fzol and the ubiquitin ligase SCF Mdm30 in mitochondrial outer membrane fusion. J Cell Sci 124: 1403-1410, 2011.
31. Cohen MM, Leboucher GP, Livnat-Levanon N, Glickman MH and Weissman AM: Ubiquitin-proteasome-dependent degradation of a mitofusin, a critical regulator of mitochondrial fusion. Mol Biol Cell 19: 2457-2464, 2008.

32. Park YY, Lee S, Karbowski M, Neutzner A, Youle RJ and Cho H: Loss of MARCH5 mitochondrial E3 ubiquitin ligase induces cellular senescence through dynamin-related protein 1 and mitofusin 1. J Cell Sci 123: 619-626, 2010.

33. Kaddour-Djebbar I, Lakshmikanthan V, Shirley RB, Ma Y, Lewis RW and Kumar MV: Therapeutic advantage of combining calcium channel blockers and TRAIL in prostate cancer. Mol Cancer Ther 5: 1958-1966, 2006.

34. Jiang S, Zu Y, Wang Z, Zhang Y and Fu Y: Involvement of mitochondrial permeability transition pore opening in 7-xylosyl-10-deacetylpaclitaxel-induced apoptosis. Planta Med 77: 1005-1012, 2011

35. Karbowski $M$ and Youle RJ: Dynamics of mitochondrial morphology in healthy cells and during apoptosis. Cell Death Differ 10: 870-880, 2003

36. Yingkun N, Lvsong $\mathrm{Z}$ and Huimin Y: Shikonin inhibits the proliferation and induces the apoptosis of human HepG2 cells. Can J Physiol Pharmacol 88: 1138-1146, 2010.

37. Knott $\mathrm{AB}$ and Bossy-Wetzel E: Impairing the mitochondrial fission and fusion balance: a new mechanism of neurodegeneration. Ann NY Acad Sci 1147: 283-292, 2008

38. Wasilewski M and Scorrano L: The changing shape of mitochondrial apoptosis. Trends Endocrinol Metab 20: 287-294, 2009.

39. Chang CR and Blackstone C: Cyclic AMP-dependent protein kinase phosphorylation of Drp1 regulates its GTPase activity and mitochondrial morphology. J Biol Chem 282: 21583-21587, 2007.

40. Cribbs JT and Strack S: Reversible phosphorylation of Drp1 by cyclic AMP-dependent protein kinase and calcineurin regulates mitochondrial fission and cell death. EMBO Rep 8: 939-944, 2007.

41. Taguchi N, Ishihara N, Jofuku A, Oka T and Mihara K: Mitotic phosphorylation of dynamin-related GTPase Drp1 participates in mitochondrial fission. J Biol Chem 282: 11521-11529, 2007.

42. Escobar-Henriques M, Westermann B and Langer T: Regulation of mitochondrial fusion by the F-box protein Mdm30 involves proteasome-independent turnover of Fzo1. J Cell Biol 173: 645-650, 2006.

43. Chan NC, Salazar AM, Pham AH, et al: Broad activation of the ubiquitin-proteasome system by Parkin is critical for mitophagy. Hum Mol Genet 20: 1726-1737, 2011.

44. Rakovic A, Grunewald A, Kottwitz J, et al: Mutations in PINK1 and Parkin impair ubiquitination of Mitofusins in human fibroblasts. PLoS One 6: e16746, 2011.

45. Nakamura N, Kimura Y, Tokuda $M$, Honda $S$ and Hirose $S$ : MARCH-V is a novel mitofusin 2- and Drp1-binding protein able to change mitochondrial morphology. EMBO Rep 7 : 1019-1022, 2006.

46. Chiche J, Rouleau M, Gounon P, Brahimi-Horn MC, Pouyssegur J and Mazure NM: Hypoxic enlarged mitochondria protect cancer cells from apoptotic stimuli. J Cell Physiol 222: 648-657, 2010

47. Ong SB, Subrayan S, Lim SY, Yellon DM, Davidson SM and Hausenloy DJ: Inhibiting mitochondrial fission protects the heart against ischemia/reperfusion injury. Circulation 121: 2012-2022, 2010. 